\title{
Malign peritoneal mesothelioma with nephrotic syndrome
}

\author{
Dogan $\mathrm{M}^{1}$, Ozal $\mathrm{G}^{1}$, Savas B², Gungor $\mathrm{M}^{3}$, Utkan $\mathrm{G}^{1}$, Yalcin $\mathrm{B}^{1}$, Icli F $\mathrm{F}^{1}$ \\ Department of Medical Oncology, Ankara University School of Medicine, Ankara, Turkey. \\ mutludogan1@yahoo.com
}

\begin{abstract}
Nephrotic syndrome may occur in malignancies like Hodgkin Disease and other solid tumors due to glomerulonephritis, amyloidosis or other causes. However, it is rare in peritoneal mesothelioma. We report a 42-year old female patient with peritoneal mesothelioma and nephrotic syndrome together with a review of literature (Tab. 1, Fig. 3, Ref. 7). Full Text in free PDF www.bmj.sk.

Key words: malign mesothelioma, peritoneal mesothelioma, nephrotic syndrome.
\end{abstract}

Malign mesothelioma (MM) is a rare neoplasm of serous membranes, like pleura, peritoneum, tunica vaginalis and albuginea. Peritoneal MM incidence in the United States of America is 2,2/1000000, and it is 1000 -fold higher in Turkey, especially in the regions, which are endemic for asbestosis, such as Kapadokya $(1,2)$.

Nephrotic syndrome (NS) may occur due to membranous glomerulonephritis, minimal change glomerulonephritis and amyloidosis in cancer patients. Ten percent of idiopathic NS cases also have cancer (3). It is uncommon in malign mesothelioma. Pleural MM patients with NS were reported in literature in early 1990s and it was shown that most of them had the minimal change glomerulonephritis $(4,5)$. However, it is very rare in peritoneal MM. In this report, we present a 42-year old female patient with NS and peritoneal MM.

\section{Case description}

A 42-year old female patient was admitted to the hospital with an abdominal distention for 2 months. Her 'Eastern Cooperative Oncology Group’ performance status (ECOG) was 2. On physical examination, she had ascites and abdominal tenderness, but did not have either defense or rebound. She had bilaterally pitting pretibial edema $(+/+)$. She was born in Corum, a city, which is rich in asbestosis, and had exposure to asbestosis for 15 years in early years of her life. She had bronchial astma, which was well controlled by avoiding precipitant factors and hypertension regulated by diet. There was no pathology on arterial blood gases. Her

${ }^{1}$ Department of Medical Oncology, Ankara University School of Medicine, Ankara, Turkey, ${ }^{2}$ Department of Pathology, Ankara University School of Medicine, Ankara, Turkey, and ${ }^{3}$ Department of Obstetric and Gynecology, Ankara University School of Medicine, Ankara, Turkey

Address for correspondence: M. Dogan, MD, Ankara University School of Medicine, Cebeci Hospital, Department of Medical Oncology, 06590 Dikimevi, Ankara, Turkey.

Phone: +903125957112, Fax: +903123192283
Tab. 1. Laboratory tests with normal values.

\begin{tabular}{lll}
\hline & Patient & Normal \\
\hline Creatinine (mg/dL) & 1.9 & $0.5-0.9$ \\
Albumin (g/dL) & 3.1 & $2.5-4.0$ \\
Total protein (g/dL) & 5.6 & $6.4-8.3$ \\
Total cholesterol (mg/dL) & 217 & $<200$ \\
LDL-cholesterol* (mg/dL) & 145.8 & $<100$ \\
HDL-cholesterol** (mg/dL) & 47 & $40-60$ \\
Triglyceride (mg/dL) & 195 & $<150$ \\
\hline
\end{tabular}

* Low density lipoprotein, ** High-density lipoprotein

creatinine level was high, and she had hypoproteinemia with hyperlipidemia on laboratory tests (Tab. 1). Her creatinine clearence was $57 \mathrm{ml} /$ minute with proteinuria (16.8 g/day). Laparoscopic biopsy of omentum revealed a tumor infiltration (Fig. 1a, 1b). There was no objective response to chemotherapy including carboplatin and gemcitabine. The patient underwent surgery for intestinal and peritoneal implants besides pouch of Douglas on intestinal explorative laparotomy, since she had symptomatic disease. There was diffuse and strong calretinin expression with keratin $5 / 6$ positivity in tumor cells on immunhistochemical staining (Figs 2 and 3). She was diagnosed with peritoneal MM. She refused renal biopsy for NS etiology. Ascites fluid was exudate with (fluid/serum) ratios as 0.34 for protein and 0.3 for lactate dehydrogenase. She received a supportive care with diet, salicylic acid and ramipril for NS. Ascites and proteinuria regressed with supportive care. Proteinuria was $1.6-2.5 \mathrm{~g} /$ day and creatinine level was $1.4 \mathrm{mg} / \mathrm{day}$. She did not need a dialysis. Although she had a chronic renal failure and NS, her renal size was not small on urinary ultrasound. Amyloidosis was ruled-out by duodenal biopsy. She has been followed-up for 9 months with a mild ascites.

\section{Discussion}

Malign mesothelioma is more common in Turkey and may be considered as one of the most important and specific diseases for 

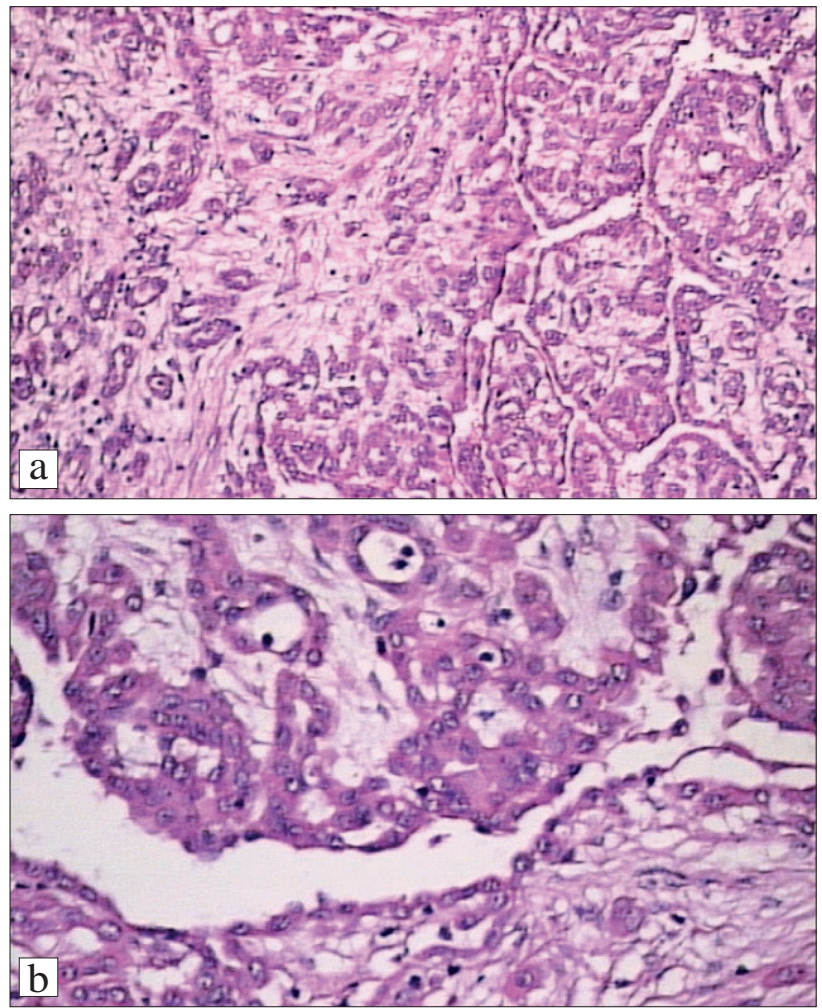

Fig. 1a,1b. Tumor infiltration in omentum (1a: HEx200, 1b: HEx400).

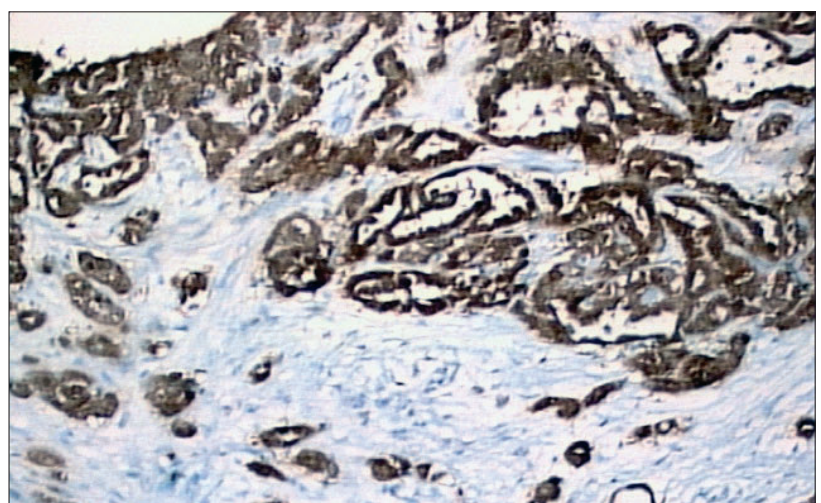

Fig. 2. Diffuse and strong calretinin expression in tumor cells (anticalretinin, $\mathrm{x} 200$ ).

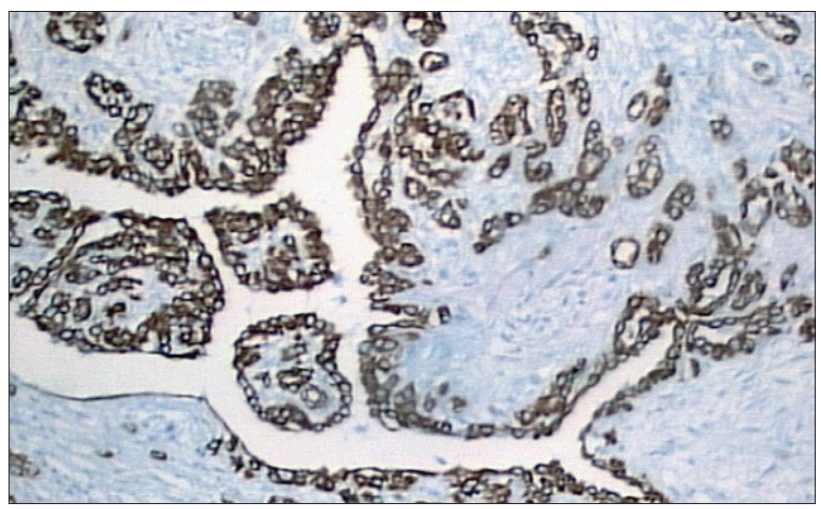

Fig. 3. Keratin 5/6 positivity in tumor cells (anti-CK5/6, x200). the country. Furthermore, we believe that discussing almost rare variants might contribute to literature.

Peritoneal MM should be kept in mind for the patients who are admitted to the hospital with ascites, especially for those living in the regions, which are endemic for asbestosis. Hypoalbuminemia is common in malignancies. Peritoneal fluid is frequently exudate in malign ascites. However, it might have transudate characteristics in the patients who have both malignancy and NS, like in our case. We have better consider NS in the patients who have anasarca edema, hyperlipidemia, hypoproteinemia and proteinuria (>3 g/day), even with pathologically documented peritoneal MM. Amiloidosis may occur secondary to chronic diseases. If a patient has a chronic renal failure (CRF) without small renal size, like our patient, we should perform biopsy to rule out amyloidosis. Duodenum, rectum, subcutaneous fat tissue and gingiva are appropriate sites for biopsy.

Renal biopsy was planned to clarify the etiologies of NS and $\mathrm{CRF}$, however she refused. Then, we performed duodenal biopsy to rule out amyloidosis. The biopsy was not compatible with amyloidosis as it was discussed before. The improvement in our patient with diet, salicylic acid and ramipril emphasizes the importance of supportive care. Furthermore, the debulking surgery, which decreased tumor load in our patient, might have also contributed to the improvement, since removing tumors may lead to regression of nephrotic pathologies related to malignancies. In conclusion, we consider that NS might have occurred as a paraneoplastic syndrome in our case.

Malign mesothelioma is moderately chemosensitive. Platin, antifolates and doxorubicin are the most effective chemotherapeutics. However, the response rates with these agents are almost lower than $20 \%$ (6).

Cytoreductive surgery and intraoperative hypertermic perfusion therapy increases the survival rates (7). Curative surgery is generally not possible in peritoneal malign mesothelioma, but debulking surgery may contribute to quality of life. A multidisciplinary approach including debulking surgery, chemotheary and radiotherapy may have better survival rates (2).

In conclusion, fluid characteristics should be evaluated in all patients who are admitted with ascites or effusions. Malign ascites is exudate. If cancer patients, especially those living in the regions which are endemic for asbestosis, have transudate ascites with hypoalbuminemia and proteinuria (>3 g/day), NS accompanying the malignancy should be considered. The supportive care for NS should be applied besides the primary cancer treatment.

\section{References}

Selçuk TS, Çöplü L, Emri S et al. Malignant Pleural Mesothelioma Due to Environmental Mineral Fiber Exposure in Turkey. Analysis of 135 cases. Chest 1992; 102: 790-796.

Piazza D, Caruso F, Scaringi S, et al. Primary Diffuse malignant peritoneal mesothelioma: case report and update of therapy. J Surg Oncol 2000; 75: 55-58.

Arnold SM, Lieberman FS, Foon KA. Paraneoplastic syndromes. 2197. In Cancer: principles and practice of oncology. In: De Vita V, Hellman S, 
Rosenberg SA(Eds). 7th ed. Philedelphia: Lippincot Williams \& Wilkins, 2005.

Venzano C, Di Marco E, Garbero M et al. Nephrotic syndrome associated with pleural mesothelioma. An unusual paraneoplastic event. Recenti Prog Med 1990; 81: 325-326.

Schroeter NJ, Rushing DA, Parker JP et al. Minimal-change nephrotic syndrome associated with malignant mesothelioma. Arch Intern Med 1986; 146: 1834-1836.
Kindler HL. Malignant pleural mesothelioma. Curr Treat Options Oncol 2000; 1: 313-326.

Sugarbaker PH, Acherman YI, Gonzalez-Moreno S et al. Diagnosis and treatment of peritoneal mesothelioma: the Washington Cancer Institute experience. Semin Oncol 2002; 29: 51-61.

Received August 10, 2009. Accepted September 20, 2011. 